\title{
SURFACE CURRENT DENSITY DISTRIBUTION MEASUREMENTS OF AN ELECTRICALLY EXPLODED FOIL VIA B-DOT PROBE ARRAY DATA INVERSION
}

\author{
E. L. Ruden, D. J. Amdahl, R. H. Cooksey, P. R. Robinson \\ Air Force Research Laboratory, Directed Energy Directorate, \\ AFRL/RDHP, 3550 Aberdeen Ave. SE, Kirtland AFB, NM 87117-5776 USA
}

F. T. Analla, D. J. Brown, M. R. Kostora, J. F. Camacho

Leidos Engineering, Inc., Albuquerque, NM 87106 USA

\section{Makhin}

TechFlow Inc., 2155 Louisiana Blvd NE, Suite 4200, Albuquerque, NM 87110-5409 USA

\section{Abstract}

Measurements are presented of the current per unit length as a function of the transverse distance from the center of a water-tamped $80 \mu \mathrm{m} \mathrm{Al}$ foil that narrows to a central width of $15.2 \mathrm{~cm}$ as it explodes into warm dense matter by Ohmic heating. Current is delivered from a $36 \mu \mathrm{F}$ capacitor bank charged to $30 \mathrm{kV}$ and discharged to a peak current of $342 \mathrm{kA}$ in $2.0 \mu \mathrm{s}$. The distribution is calculated by the linear regularized inversion of signals from an array of B-dot probes distributed long the foil's central half-width. The probes are far enough away from the foil $(1 \mathrm{~cm})$ to be noninvasive and mechanically undisturbed during the time of interest. These results are compared to 3-D MHD ALEGRA simulations of the geometry externally coupled to a two-loop lumped circuit model representing the driver. Current is strongly peaked at the foil edges for low-current calibration tests, where conductivity is essentially constant. It's broadly peaked in the center at peak current for the full energy shot, though, due to the foil fusing first at the edges, which subsequently reduces current there relative to the center by the time of peak current. There is broad agreement in this regard between the experiment and ALEGRA using thermal and electrical conductivity model SNL Sesame 293731. ALEGRA peaks $0.5 \mu$ s earlier, though, and with $50 \mathrm{kA}$ higher current. This may be due to error in the conductivity tables or effects not well-modeled, such as an electro-thermal instability that results in higher total resistance, but with a distance scale too small for the present simulation to represent.

\section{INTRODUCTION}

This paper details one diagnostic of an experiment to provide the basis for 3-D modeling of transition from room $\mathrm{T}$ to plasma for full (cold start) predictive capability. The experiment produces homogeneous warm dense matter from Ohmically exploded tamped foils in the regime characteristic of single-shot pulsed power, as traced in Fig. 1. The goal, then, is to diagnose dynamic surface conditions to determine electrical coductivity and equation of state properties to validate theoretical ab initio models of matter encountered in single-shot pulsed power devices.

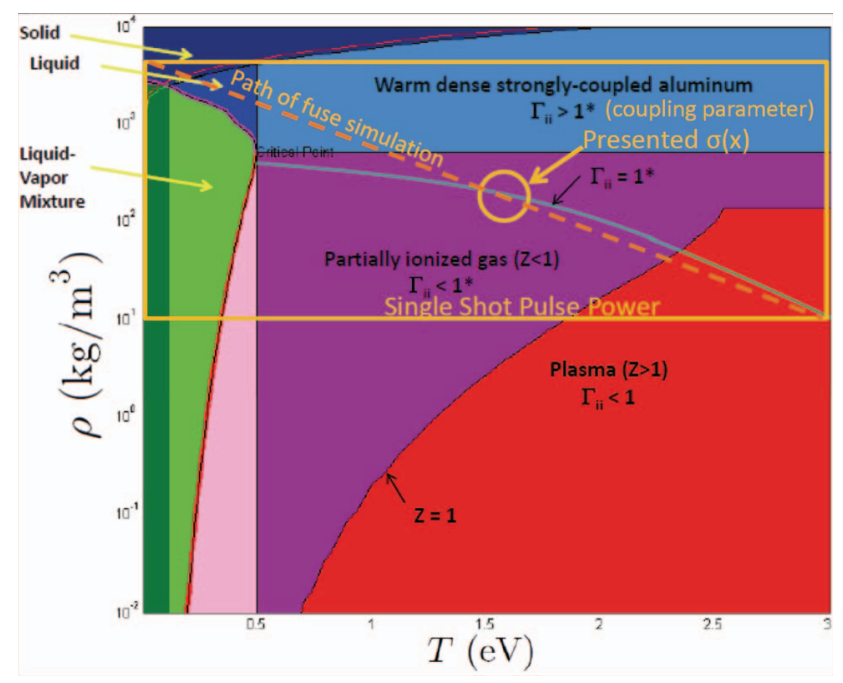

Figure 1. Phase diagram of Al Sesame table 3720 used for ALEGRA simulations.

Figure 2 is an exploded view of the load assembly. The $z$-axis is taken to be the centerline of the current density distribution, the $y$-axis passes perpendicularly through the center of the fuse, and the $x$-axis crosses the fuse's width at the center. Foil boundaries are described by

$$
\begin{gathered}
\pm x=A+B z^{2}+C z^{4} \quad A=7.62 \mathrm{~cm} \\
B=0.0117 \mathrm{~cm}^{-1} \quad C=-1.118 \times 10^{-6} \mathrm{~cm}^{-3}
\end{gathered}
$$

The subject diagnostic is an array of B-dot probes spanning the central fuse half-width at the distance of the return conductor, as shown in Fig. 3. This provides the data needed to infer surface current density distribution which, in conjunction with a dynamic fuse width mea- 
surement (not discussed in this paper), allows the mean bulk current density to be determined from the ratio.

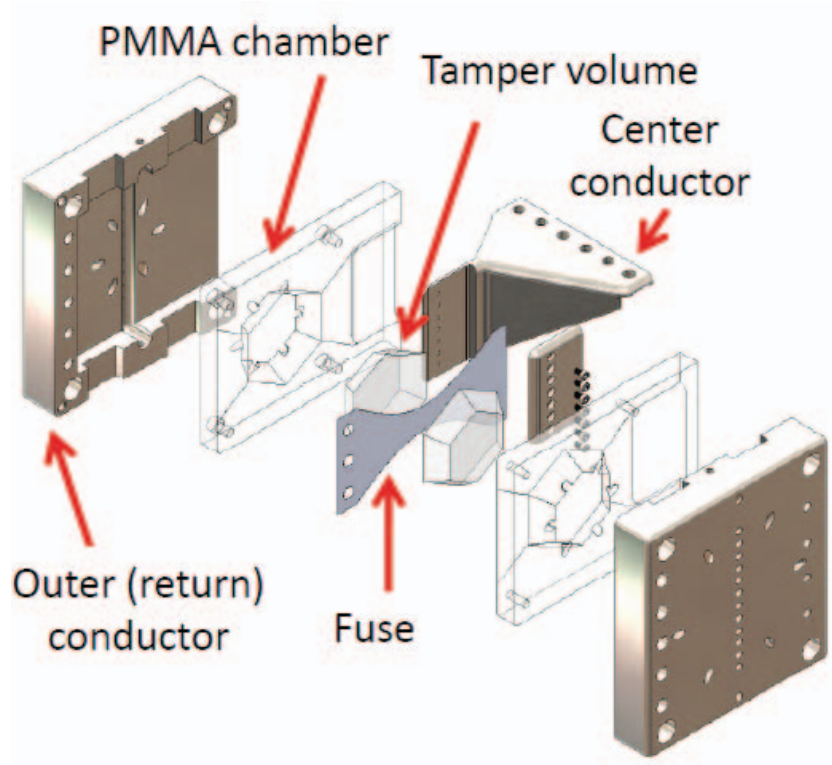

Figure 2. Exploded view of load assembly. Current is passed through central tapered fuse and symmetrically returns via encapulating conductor half-shells. The tamper volume for the presented data is filled with deionized water.

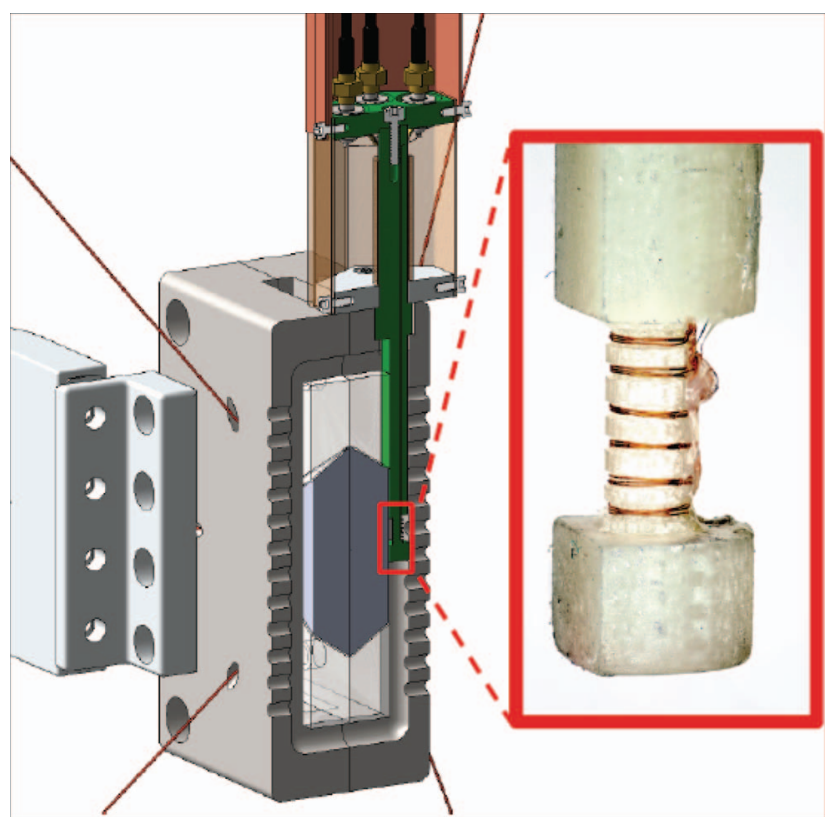

Figure 3. Load assembly cut-away through $x-y$ plane passing through fuse center ( $z$-axis is current path centerline). A 6-head B-dot probe array is inserted into a semicircular channel in one return conductor half-shell.

\section{DATA INVERSION THEORY}

An array of $p$ B-dot probes flush with the return conductor near the load center, as shown in Fig. 4, is used to infer the current per unit width $\sigma(x)$ across the foil at its narrowest point. To calibrate the response of the probes to the current distribution, the foil is replaced for $q$ tests with a series of wire pairs bent to the theoretical shape of current streamlines, based on a low energy ALEGRA simulation. The sensitivity of each probe to fuse current following each streamline is then determined. For each calibration test, $I_{j}$ is the measured current in wire pair $j$ which passes through the load center at $x= \pm x_{j}$ where,

$$
x_{j}=\left(j-\frac{1}{2}\right) \Delta x \quad j=1 \ldots q
$$

Here, $q \Delta x$ is the foil central width $W$. The contribution to the time integrated signal $B_{i}$ of probe $i$ from wire pair $j$ is $M_{i j} I_{j}$, where,

$$
M_{i j}=\frac{\text { integrated B-dot signal for probe } i}{\text { current waveform signal for wire pair } j}
$$

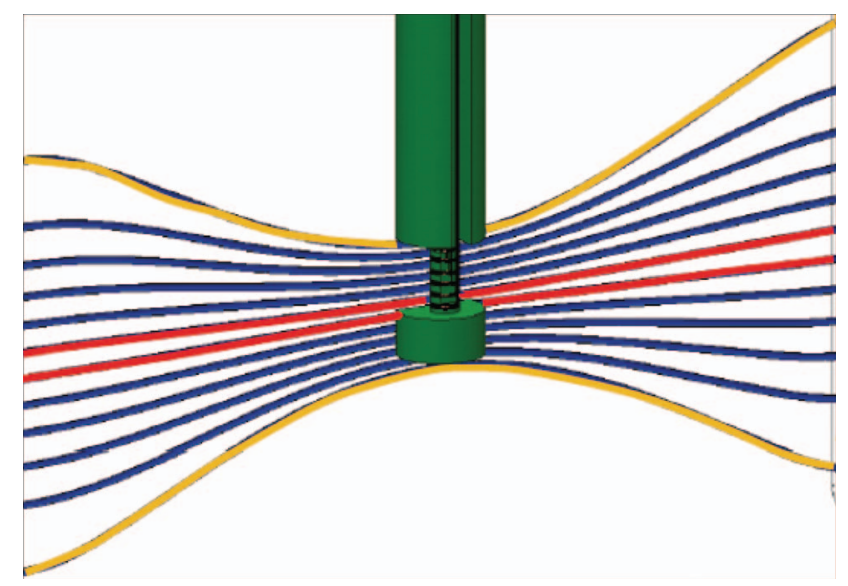

Figure 4. The fuse surface current density distribution is decomposed into 6 current element pairs assumed to follow the current density streamlines of a low-energy ALEGRA simulation and represented by actual wires on the calibration stand, as drawn here. A 6-head B-dot probe assembly (green) within a channel in the return conductor spans the central fuse half-width, but separated from it by the tamper.

From the principle of superposition, the set of $p$ time integrated B-dot signals $(\mathrm{V} \cdot \mathrm{s})$, represented by column vector $\mathbf{B}$ is,

$$
\begin{array}{cc}
\mathbf{B}=\mathbf{M I}, & \mathbf{I} \in \Re^{q} \\
\mathbf{M} \in \Re^{p \times q}, & \mathbf{B} \in \Re^{p}
\end{array}
$$

where $\mathrm{M}(\mathrm{V} \cdot \mathrm{s} / \mathrm{A})$ is the $\mathrm{B}-\mathrm{dot}$ probe calibration matrix, and $\mathbf{I}$ (A) is the column vector of the $q$ current elements representing the surface current density distribution. The inverse problem of inferring $\mathbf{I}$ (and therefore $\sigma(x)$ ) from 
B measured during an actual fuse test, then, must be solved.

Data inversion is accomplished via linear regularization[1]. For this, an approximation to $\mathbf{I}$ is found which minimizes,

$$
\begin{gathered}
|\mathbf{M I}-\mathbf{B}|^{2}+\lambda|\mathbf{D I}|^{2} \\
\mathbf{D} \in \Re^{r \times q}, \quad r \leq q
\end{gathered}
$$

where $\mathbf{D}$ is a matrix operator characterizing the high spatial frequencies ("noise") we wish to suppress (typically a spatial derivative), $\lambda$ is the weighting factor for such, $|\mathbf{X}|^{2}=\mathbf{X}^{\mathrm{T}} \mathbf{X}$ (scalar magnitude squared) if $\mathbf{X}$ is a column vector (as is the case above), and superscipt $\mathrm{T}$ means transpose. $\lambda$ may be expressed as

$$
\lambda=\alpha \frac{\operatorname{Tr}\left(\mathbf{M}^{\mathrm{T}} \mathbf{M}\right)}{\operatorname{Tr}\left(\mathbf{D}^{\mathrm{T}} \mathbf{D}\right)}
$$

where $\alpha$ generally ranges from 0 for no noise suppression to a number of order 1 for strong suppression.

I is the solution to

$$
\left(\mathbf{M}^{\mathrm{T}} \mathbf{M}+\lambda \mathbf{D}^{\mathrm{T}} \mathbf{D}\right) \mathbf{I}=\mathbf{M}^{\mathrm{T}} \mathbf{B}
$$

which is solved with the help of singular value decomposition[2] of the term in (). That is, $\mathbf{U}, \mathbf{W}$ and $\mathbf{V}$ are found (by MATLAB, in our case) such that,

$$
\left(\mathbf{M}^{\mathrm{T}} \mathbf{M}+\boldsymbol{\lambda} \mathbf{D}^{\mathrm{T}} \mathbf{D}\right)=\mathbf{U} \mathbf{W} \mathbf{V}^{\mathrm{T}}
$$

where $\mathbf{U}$ and $\mathbf{V}$ are orthogonal, and $\mathbf{W}$ is a diagonal matrix with nonnegative elements,

$$
\begin{gathered}
\mathbf{U}^{\mathrm{T}} \mathbf{U}=\mathbf{1}, \quad \mathbf{V}^{\mathrm{T}} \mathbf{V}=\mathbf{V V}^{\mathrm{T}}=\mathbf{1} \\
\mathbf{W}=\operatorname{diag}\left(w_{1}, \ldots, w_{q}\right), w_{j+1} \geq w_{j}
\end{gathered}
$$

Here, $\mathbf{1}$ is the $q \times q$ identity matrix. The solution then is,

$$
\begin{gathered}
\mathbf{I}=\mathbf{V} \mathbf{W}^{-1} \mathbf{U}^{\mathrm{T}} \mathbf{M}^{\mathrm{T}} \mathbf{B} \\
\mathbf{W}^{-1}=\operatorname{diag}\left(w_{1}^{-1}, \ldots, w_{q}^{-1}\right)
\end{gathered}
$$

Per method, if any of the $\left\{w_{i}\right\}$ values are zero (or, in practice, very small), the corresponding diagonal element of $\mathbf{W}^{-1}$ is set to zero.

Order 2 regularization (based on the second spatial derivative) is used here. We modify Press's D matrix[1] (Section 18.5) for this by taking as a priori that $\mathbf{I}$ represents a distribution symmetric about $x=0$. To this end, without modifying the definition of $\mathbf{I}$ (it will remain a $q$-element column vector), we define extra elements external to $\mathbf{I}$ with currents $I_{0}=I_{1}$ and $I_{-1}=I_{2}$. These correspond to "ghost" elements at $x=-\Delta x / 2$ and $-3 \Delta x / 2$, respectively. The second derivatives terms at $x=x_{1}$ and $x=x_{2}$ are, then, $\left(I_{-1}-2 I_{0}+I_{1}\right)=$ $\left(I_{0}-2 I_{1}+I_{2}\right)=\left(-I_{1}+I_{2}\right)$, respectively. These alter the first two rows of $\mathbf{D}$. The rest of the elements are unaltered from Press (except for a sign preference). For $q=6$, the case that we will consider, we have,

$$
\mathbf{D}=\left[\begin{array}{rrrrrr}
-1 & 1 & 0 & 0 & 0 & 0 \\
-1 & 1 & 0 & 0 & 0 & 0 \\
1 & -2 & 1 & 0 & 0 & 0 \\
0 & 1 & -2 & 1 & 0 & 0 \\
0 & 0 & 1 & -2 & 1 & 0 \\
0 & 0 & 0 & 1 & -2 & 1
\end{array}\right]
$$

Once calculated, the continuous function $\sigma(x)$ is inferred from $\mathbf{I}$ by fitting the results $\left\{x_{j}, I_{j}\right\}$ to a polynomial. We use $a x^{6}+b x^{4}+c x^{2}+d$ for the presented data. Only even powers are $x$ used here since it represents a distribution presumably symmetric about $x=0$. The polynomial is then rescaled such that its integral from $x=-W$ to $W$ equals $\sum I_{j}$ (the total current inferred from the inversion).

\section{B-DOT PROBE INTEGRATION AND CALIBRATION MATRIX}

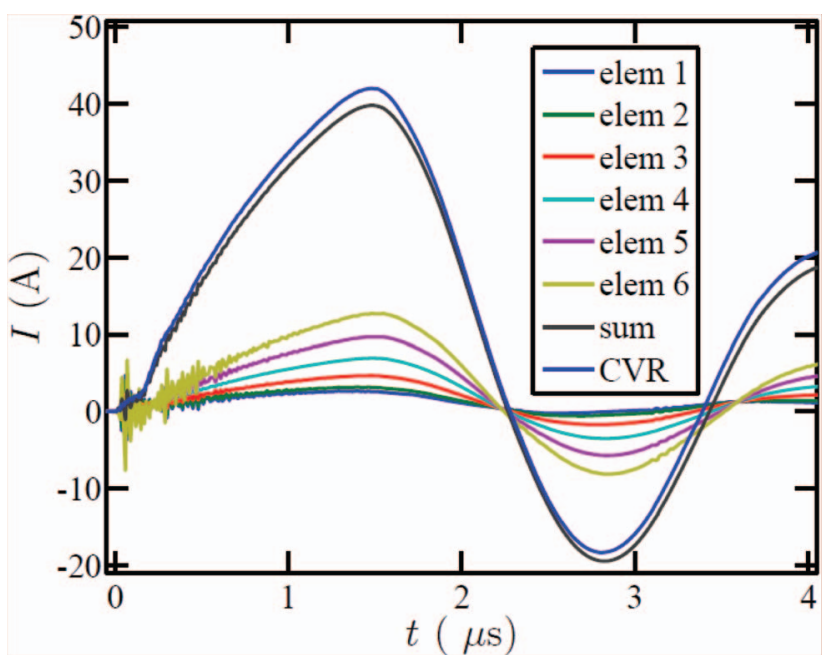

Figure 5. Element currents are plotted here resulting from multiplying the regularized inverse (with $\alpha=0.1$ ) of the calibration matrix by the integrated B-dot probe signals with a full fuse foil in the calibration stand. As a consistency check, the sum of the element currents is compared to the current directly measured with a current viewing resistor in series with the load.

The ratio of the slope of the initial baseline-corrected B-dot probe time integral at its first zero crossing to that of the recorded current trace was chosen for the matrix elements of Eq. 3. This metric minimizes the effects of early time noise and skin current. $\alpha=0.1$ was chosen as the smallest necessary to avoid numerical artifacts. Figure 5 plots the results of a test on the calibration stand where an actual load foil is installed instead of a wire pair. Figure 6 plots the surface current density distribution inferred. The original element current, rescaled by the 
same factor as the polynomial fit to be consistent with the total current, is shown as a symbol on or near each curve. The B-dot probe data taken with wire pairs 1 and 6 are also inverted using the same matrix. The smooth traces (vs spikes at each wire) illustrate that the method as implemented only provides valid results for slowly varying distributions.

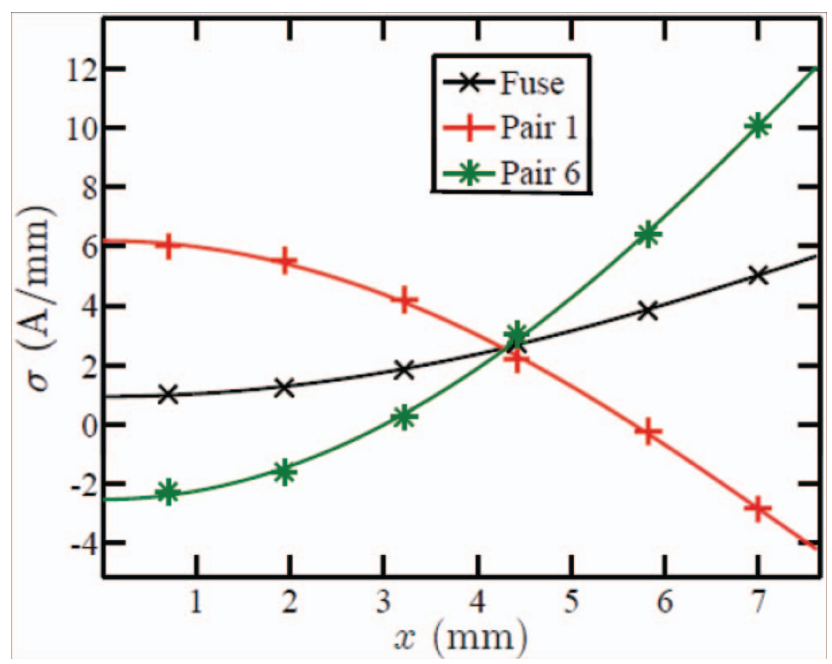

Figure 6. Surface current density vs. $x$ across the width of the fuse center inferred from the current element traces plotted in Fig. 5 at peak current. As a consistency test, the regularized inverse of the original B-dot probe signals for the calibration tests using wire pairs 1 and 6 are inverted.

\section{HIGH ENERGY EXPERIMENT}

The component values of the external coupled circuit model used by ALEGRA (Fig. 7) are iterated to accurately describe experimental waveforms of two complementary test shots. One test used a $914 \mu \mathrm{m}$ thick "fuse", which maintained near room temperature during the discharge, and so can be approximated as having a constant skin resistance. The other used an $80 \mu \mathrm{m}$ foil that opened early enough such that the circuit rang thereafter as a single loop providing complementary information about the parallel path installed to protect the capacitor bank when this happens. The results are:

$$
\begin{array}{ll}
V_{0}(0)=0.98 \times V_{00} \text { actual } \\
R_{1}=12.4 \times 10^{-4} \Omega & L_{1}=4.503 \times 10^{-8} \mathrm{H} \\
R_{2}=30.44 \times 10^{-3} \Omega & L_{2}=5.190 \times 10^{-7} \mathrm{H} \\
R_{3}=3.802 \times 10^{-3} \Omega & L_{3}=3.099 \times 10^{-8} \mathrm{H}
\end{array}
$$

Such tests determine only total thick-fuse branch values $L_{1}=L_{1 \mathrm{X}}+L_{1 \mathrm{~A}}$ and $R_{1}=R_{1 \mathrm{X}}+R_{1 \mathrm{~A}}$. The $\mathrm{X}$ (external) terms used for ALEGRA's external couple circuit model are determined by subtraction of A (ALEGRA) terms determined directly by simulation of the low resistance load setup driven at the $100 \mathrm{kHz}$ characteristic of the discharge. This simultaneously corrects for errors due to simpification of the current feed in the simulation. Also, for a better fit to the experiment, and effective initial bank charge of $0.98 \times V_{00}$ is used in the model, where $V_{00}$ is actual measured bank voltage. This is thought to compensate for the voltage-lowering effect of the capacitor bank transmission line, which is not otherwise considered. Figure 8 plots the results of the measured currents during the discharge, for which $V_{00}=30 \mathrm{kV}$. Figure 9 plots the results of B-dot probe data inversion at peak $I_{1}(2.0$ $\mu \mathrm{s})$. Increased resistivity, especially at the edges where initial current density is highest, has caused the surface current density distribution to level off considerably, and even concentrate somewhat in the center.

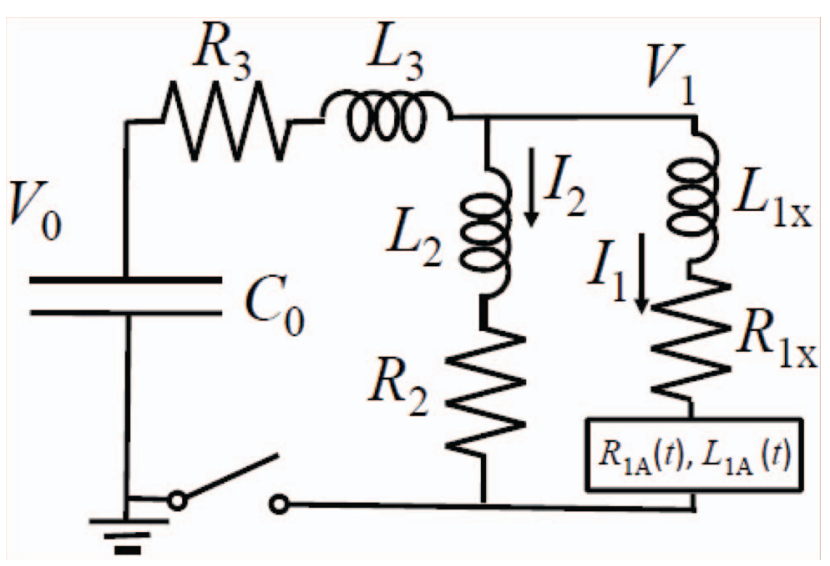

Figure 7. External circuit model coupled to ALEGRA high-energy simulation. Component values are determined empirically via complementary discharges, with theoretical contributions from the ALEGRA mesh itself (subscript A) subtracted.

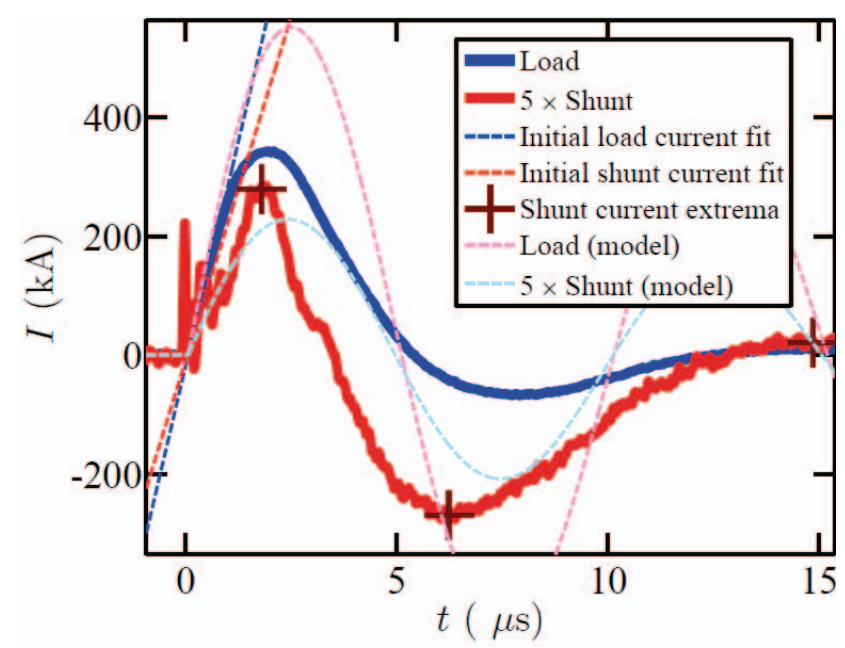

Figure 8. High energy shot load and 5 times the shunt current of the subject shot (solid lines). The two-loop model (Fig. 7) waveforms if the fuse had remained in its initial high conductivity state are overlaid. 


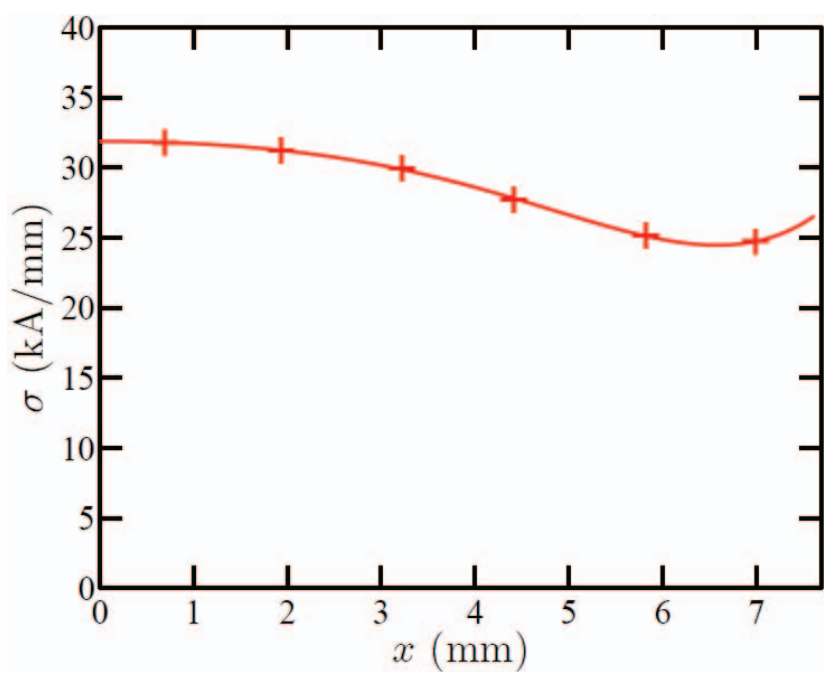

Figure 9. Surface current density vs. $x$ across the width of the fuse center at peak current inferred from B-dot measurements of the subject high energy shot using the same regularized inverse of the calibration matrix used for Fig. 5 and the same polynomial fit order used for Fig. 6 .

\section{ALEGRA SIMULATIONS}

ALEGRA, a parallel 3D resistive MHD code[3], is used to estimate plasma properties and surface current density (by integration of current density) of the fuse. Three runs are performed at the Department of Defense high performance computing center at the Army Research Laboratory using the geometry of Fig. 10 as the load coupled via a potential solve boundary condition to either a predefined current or an externally coupled circuit. Approximately 940,000 cells on 128 processors are used.

The first run drives the fuse with the current defined by that measured for the calibration data for comparison with that test. For the second, the load is driven at a low current at $100 \mathrm{kHz}$ to determine the "A" terms of Fig. 7 for subtraction from the empirical totals of Eqs. 12. The results are $R_{1 \mathrm{~A}}=2.1 \times 10^{-3} \Omega$ and $L_{1 \mathrm{~A}}=18.335 \times 10^{-9}$ H. Note that $R_{1 \mathrm{~A}}$ here is larger that $R_{1}$, requiring $R_{1 \mathrm{X}}$ to be negative. This is an artifact of the ALEGRA interface being much longer and simpler than the actual convolution to simplify meshing. The interface is assumed, though, to only significantly affect the load due to its contribution to $R_{1}$ and $L_{1}$. The overestimate is compensated for by adjusting the external contributions accordingly. The third run simulates the high energy experiment with the full externally coupled circuit charged to the same initial voltage, but free to react based on the simulated dynamic load impedance. Figures 11-15 plot the results of the latter two runs.

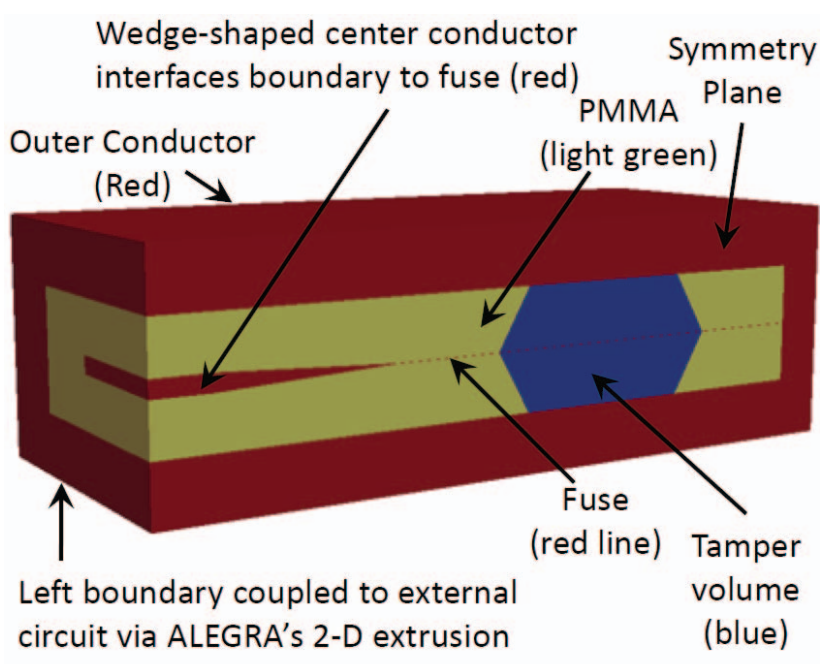

potential solver. For high-energy shot.

Figure 10. Load geometry simulated by ALEGRA.

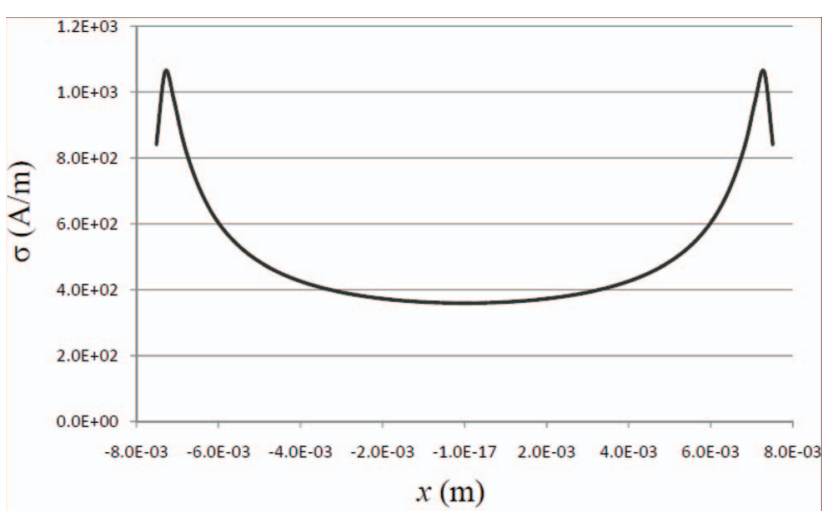

Figure 11. Surface current density vs. $x$ across the width of the fuse center at peak current as calcuated by ALEGRA assuming the defined input current of Fig. 5 measured for the calibration tests, for comparison with experiment (Fig. 6).

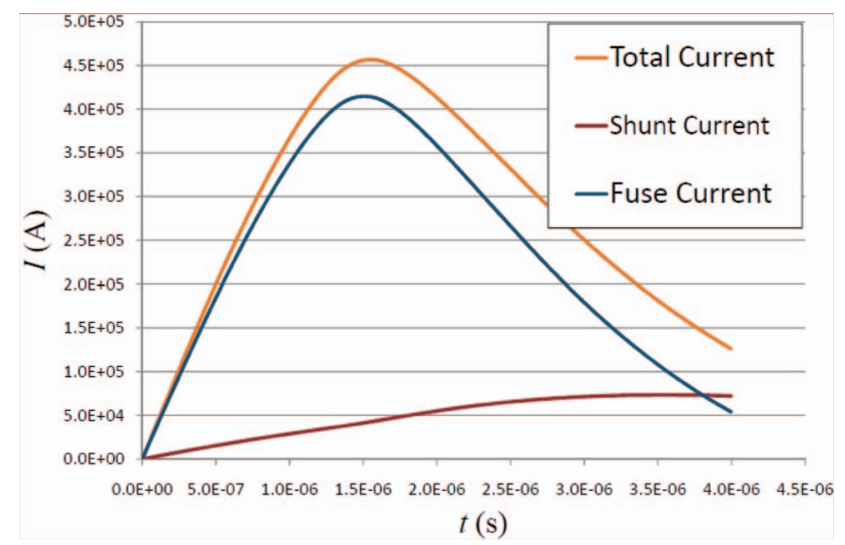

Figure 12. Currents $I_{1}$ (fuse) and $I_{2}$ (shunt) and $I_{1}+$ $I_{2}$ of external coupled circuit for high energy ALEGRA simulation. 


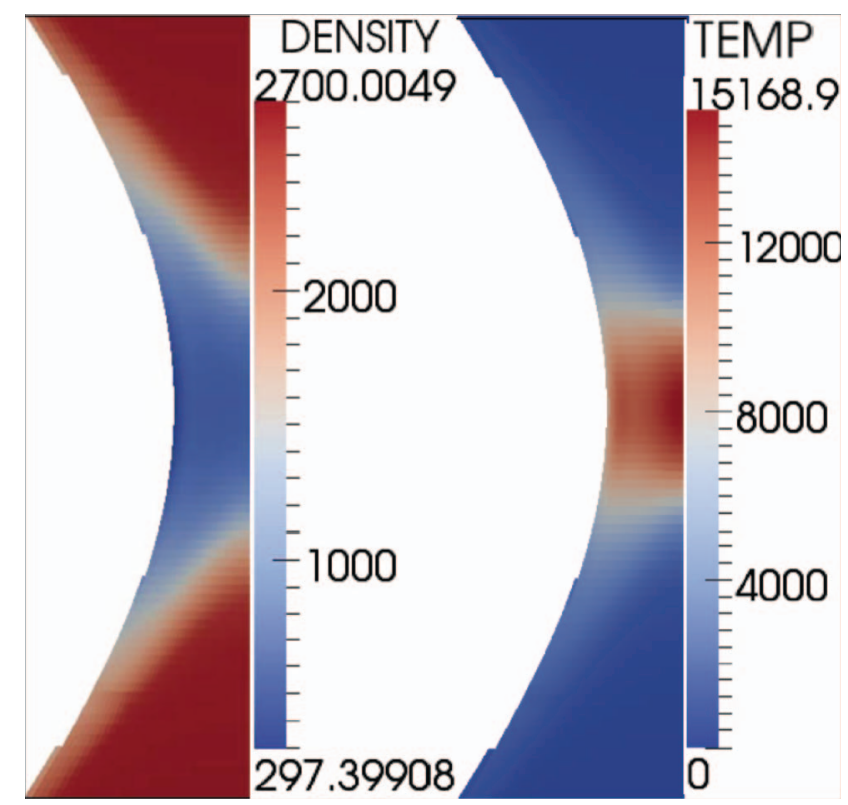

Figure 13. Fuse density $\left(\mathrm{kg} / \mathrm{m}^{3}\right)$ and Temperature $(\mathrm{K})$ at fuse midplane for high energy ALEGRA simulation near peak current $(t=15 \mu \mathrm{s})$.

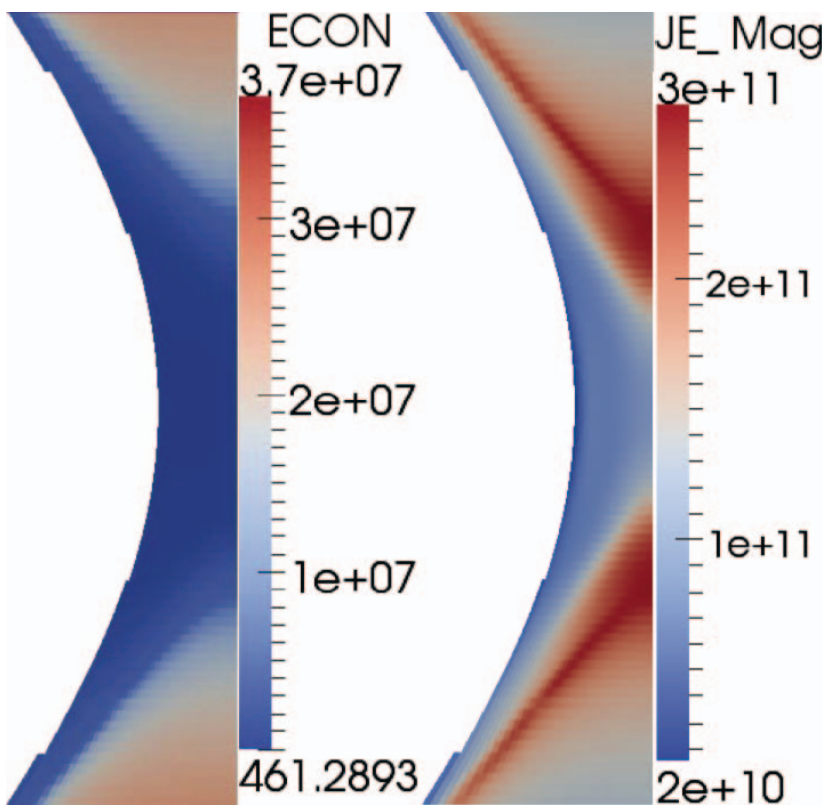

Figure 14. Fuse electrical conductivity $(\mathrm{S} / \mathrm{m})$ and current density magnitude $(\mathrm{A} / \mathrm{m})$ at fuse midplane for high energy ALEGRA simulation near peak current $(t=15 \mu \mathrm{s})$.

Data employed for Al are SNL Sesame 3720 (EOS); Steinberg Guinan Lund 6061-T6 (strength); SNL Sesame 29373 with 601 (ionization state), 602 (electrical conductivity), and 603 (thermal conductivity). Data for water are Sesame EOS KEOS , matlabel = "water", neos = 7150; no strength; thermal conductivity $=0.58 \mathrm{~W} /(\mathrm{m} \mathrm{K})$, electrical conductivity $=5 \times 10^{-3}(\mathrm{~S} / \mathrm{m})$. Data for PMMA are Sesame EOS KEOS Mie Gruneisen, matlabel = "pmma", neos $=7150$; Steinberg Guinan Lund (strength); thermal conductivity $=0.25 \mathrm{~W} /(\mathrm{m} \mathrm{K})$; electrical conductivity $=1 \times 10^{-7}(\mathrm{~S} / \mathrm{m})$.

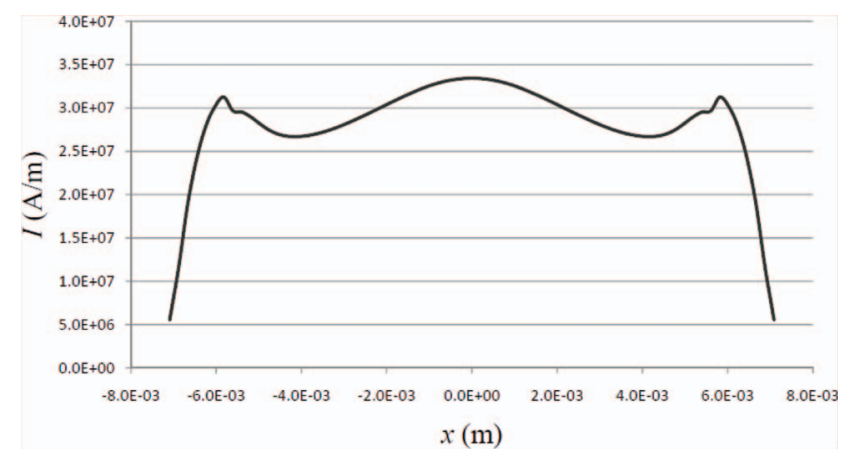

Figure 15. Surface current density vs. $x$ across the width of the fuse center near peak current $(t=15 \mu \mathrm{s})$ for comparison with experiment (Fig. 8).

\section{CONCLUSIONS}

The calibration fuse current, with its essentially uniform conductivity, is greatest at the edges both experimentally and in the simulation, although regularization in the former does not allow for sufficient spatial resolution to show it as sharply as ALEGRA. For the high energy shot, though, it's broadly peaked in the middle. This occurs due to the foil fusing at the edges first, subsequently reducing current there. There is broad agreement between ALEGRA and the experiment in this regard too. ALEGRA, though, peaks $0.5 \mu$ s earlier, and with $50 \mathrm{kA}$ higher current. This may be due to error in the conductivity tables or other physics not captured in the simulation. For example, electro-thermal instabilities on a distance scale too small for the present simulation to capture may result in higher total resistance.

\section{REFERENCES}

[1] W. H. Press, S. A. Teukolsky, W. T. Vetterling, and B. P. Flannery, Numerical Recipes in C, 2nd ed. New York, NY: Cambridge Univ. Press, 1992.

[2] G. H. Golub and C. F. Van Loan, Matrix Computations, New York, NY: John Hopkins Univ. Press, 1983.

[3] A. C. Robinson, et al., "ALEGRA: An arbitrary Lagrangian-Eulerian multimaterial, multiphysics code," 46'th AIAA Aerospace Sciences Meeting and Exhibit, 710 Jan 2008, Reno, NV, 2008, AIAA 2008-1235. 